\title{
Analysis of adaptive foraging in an intraguild predation system
}

\author{
Toshinori Okuyama and Robert L. Ruyle
}

Okuyama, T. and Ruyle, R. L. 2003. Analysis of adaptive foraging in an intraguild predation system. - Web Ecol. 4: 1-6.

\begin{abstract}
An intraguild predation (IGP) system with adaptive foraging behavior was analyzed using a simple mathematical model. The main aim was to explore how the adaptive behavior affects species interactions as well as how such interactions derived from adaptive behavior affect community stability. The focal system contained top predators, intermediate predators, and basal prey. Intermediate predators exhibit antipredator behavior and balance costs (e.g., perceived predation risk) and benefits (e.g. resource intake) to determine their foraging effort. Density-dependent foraging behavior with the unique connectance of the IGP food web created unusual species interactions. Notably, increased prey density can transmit negative indirect effects to top predators while increased top predator density transmits positive indirect effects to prey population. The nature of these interactions is density-dependent. The results suggest that both IGP (as opposed to linear food chain) and adaptive foraging behaviors may strongly influence community dynamics due to emergent interactions among direct effects and indirect effects. Furthermore, the adaptive foraging of intermediate predators may stabilize the community as a whole.
\end{abstract}

T. Okuyama (toshi@zoo.ufl.edu), School of Biological Sciences, Univ. of Nebraska-Lincoln, Lincoln, NE 68688-0118, USA, present adress: Zoology Dept, Univ. of Florida, Gainesville, FL 32611-8525, USA. - R. L. Ruyle, Dept of Mathematics and Statistics, Univ. of Nebraska-Lincoln, Lincoln, NE 68588-0323, USA.

Although intraguild predation (IGP) is well recognized as an important source of complexity in applied settings as in agriculture and conservation (Polis and Holt 1992, Fagan et al. 1998, Muller and Brodeur 2002, Snyder and Ives 2003) as well as ecological community in general, we lack a fundamental understanding of IGP (reviews in Polis et al. (1989) and Polis and Holt (1992)). For example, a general theory suggests that persistence of communities with IGP through ecological time scale is unlikely (Holt and Polis 1997) despite the fact IGP is ubiquitous (Suwa 1986, Polis and McCormick 1987, Szeinfeld 1991, Wissinger 1992, Johnson 1993, Moran and Hurd 1994, Dinter 1998, Lucas et al. 1998). Though not the only possible outcome, the exclusion of intermediate predators is a likely result due to the additional mortality imposed by IGP on theoretical grounds.

In order to explain this discrepancy, various hypotheses (e.g. stage-structured population (Borer 2002), adaptive behaviors (Křivan 2000), spatial heterogeneity (Holt and Polis 1997), and spatial refugia (Sergio et al. 2003)) have been proposed. However, most of these factors have not been explicitly incorporated in IGP theory. Among the proposed hypotheses, adaptive foraging behavior has been studied both within and outside the IGP framework, and results from those studies indicate that such behavior may be an important mechanism that allow many complex ec- 
ological systems to exist (Kondoh 2003). Křivan (2000) studied the effects of optimal foraging in an IGP system in which the top predators optimize the proportion of prey types (intermediate predators and basal prey) eaten and showed that adaptive intraguild predation is a stabilizing factor provided environmental productivity is high.

In addition to theoretical support for the importance of adaptive foraging behaviors, there is compelling empirical evidence that antipredator behavior (one of the most prevalent adaptive behavior) is quantitatively important in many food webs (Schmitz 1998, Okuyama 2002). One important aspect of antipredator behavior in food webs is that it creates trait-mediated indirect interactions. Such interactions are transmitted by qualitative changes in the traits of intervening species and are known to alter structure of many communities (Werner and Peacor 2003, Bolker et al. 2003). Antipredator behavior is one of the most widespread factors responsible for the prominence of trait-mediated indirect interactions in nature.

In this paper, we consider the roles of adaptive foraging of intermediate predators in a system of IGP. The aim of the current paper is to 1) characterize the interaction of various direct effects and indirect (both trait-mediated and density-mediated) effects, and 2) examine the effect of such behavior on the stability of the community.

\section{IGP system}

We considered the simplest IGP system where there are top predators, intermediate predators, and basal prey (Fig. 1). Top predators and intermediate predators share the basal prey as a food resource while top predators also prey on

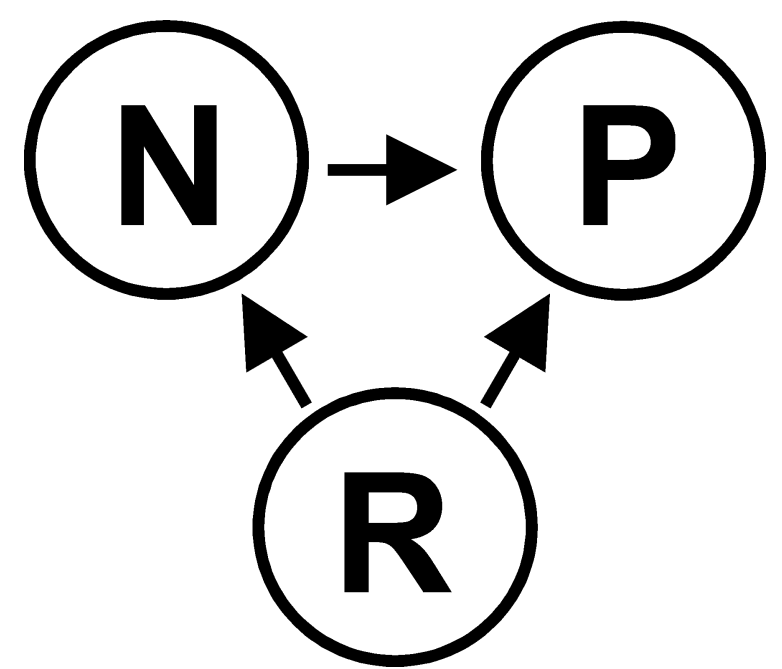

Fig. 1. The simple IGP system. P (top predators), N (intermediate predators) and $\mathrm{R}$ (basal prey). The direction of the arrows indicates the energy flow (e.g. P eats R). intermediate predators. As a result, intermediate predators play the roles of both predators and prey. In other words, intermediate predators and top predators experience exploitative competition by consuming a common resource while they also maintain a predator-prey relationship. In addition, this food web creates a loop connecting the three species, which allows all species to influence one another through both indirect and direct means.

\section{Adaptive foraging model}

For the analysis of this model, we followed the technique described by Abrams (1992). In the IGP system described above, intermediate predators exhibit antipredator behavior. Top predators are predation-free, and they are assumed to forage at their maximum capability. We also assume that basal prey do not exhibit adaptive foraging behavior, meaning the presence of predators does not alter their foraging behavior. This assumption is reasonable when the basal prey are sessile or not very vigilant (e.g. Okuyama 2002). The foraging effort of intermediate predators is denoted by $\mathrm{C}_{\mathrm{N}}$, which can be considered as a foraging activity, such as foraging time, that directly influences the risk of predation and resource intake. Intermediate predators increase their resource gain by increasing foraging effort, while increasing foraging effort also increases their risk of predation. The vulnerability function for intermediate predators, $\mathrm{f}_{\mathrm{N}}\left(\mathrm{C}_{\mathrm{N}}\right)$, is also an increasing function of the foraging effort. Foragers alter their foraging effort by balancing the associated costs and benefits in order to maximize their fitness. Fitness is expressed as the difference between an instantaneous per capita birth rate and death rate. The death rate is the sum of deaths from predation and deaths due to other causes. Under these assumptions, the system of equations can be expressed as follows:

$$
\begin{aligned}
& \frac{d P}{d t}=P\left(B_{P}\left(R+f_{N} N\right)-m_{P}\right) \\
& \frac{d N}{d t}=N\left(B_{N}\left(C_{N} R\right)-f_{N} P-m_{N}\right) \\
& \frac{d R}{d t}=R\left(\Phi-C_{N} N-P\right)
\end{aligned}
$$

where $\mathrm{P}, \mathrm{N}$ and $\mathrm{R}$ are the densities of top predators, intermediate predators, and basal prey, respectively. $B_{P}$ and $B_{N}$ scale the benefit experienced by top predators and intermediate predators from their resource(s), respectively. $m_{P}$ and $\mathrm{m}_{\mathrm{N}}$ are the density-independent mortality rates of top predators and intermediate predators respectively. $\Phi$ is the recruitment of basal prey.

\section{Analysis}

Abrams (1992) analyzed the model in two different scenarios: the case where the benefit functions, $\mathrm{B}_{\mathrm{P}}$ and $\mathrm{B}_{\mathrm{N}}$, are 
linear and the vulnerability function, $\mathrm{f}_{\mathrm{N}}$, is nonlinear, and the reverse case where the benefit functions are nonlinear and the vulnerability function is linear. The latter case is more realistic as there are constraints on any animal's rate of converting captured resource into offspring (Abrams 1992). Hence, in our study, we only considered the case where benefit functions are nonlinear and the vulnerability function is linear. The nonlinear foraging benefits suggest that eating particular amounts of prey may increase their benefit comparatively rapidly or little depending on the nonlinearity. Consequently, as prey population size increases, predators may need to expend only so much effort to obtain desirable benefits, which may result in decreased foraging effort. A linear vulnerability function, $\mathrm{f}_{\mathrm{N}}$, means that there is a constant risk per unit foraging effort. Therefore, if a forager spends twice as much effort, it will incur twice the predation risk. According to these assumptions, the food chain descriptions can be shown as follows:

$$
\begin{aligned}
& \text { Top predator fitness }=W_{P}=B_{P}\left(R+C_{N} N\right)-m_{P} \\
& \text { Intermediate predator finess } \\
& =W_{N}=N\left(B_{N}\left(C_{N} R\right)-f_{N} P-m_{N}\right) \\
& \text { Prey fitness }=W_{R}=\Phi-C_{N} N-P .
\end{aligned}
$$

The implicit equations for optimal effort $\mathrm{C}_{\mathrm{N}}$, becomes

$$
\mathrm{B}_{\mathrm{N}}{ }^{\mathrm{R}} \mathrm{R}-\mathrm{P}=0 \quad\left(\mathrm{~B}_{\mathrm{N}}{ }^{\prime}<0\right) .
$$

To examine the effect of density-dependence on the fitness of each species due to other species, we need to know how the foraging effort of intermediate predators, $\mathrm{C}_{\mathrm{N}}$, depends on the population density of each species. This can be obtained by implicit differentiation of eq. (7). The formulae for the effects of population density on fitness are presented in Appendix 1.

The signs of the resulting interactions are shown in Table 1 , which indicates that 1 ) top predators are always negatively influenced by their own population growth 2) the effect of basal prey population on itself and on top predator population can be positive or negative depending on density 3) top predator population can both positively and negatively influence basal prey population depending on density. The sign of $\partial / \partial \mathrm{C}_{\mathrm{N}}\left(\mathrm{C}_{\mathrm{N}} \mathrm{B}_{\mathrm{N}}\right)$ determines whether or not intermediate predators increase or decrease their foraging effort in response to increased basal prey density (Abrams 1989).

\section{The mechanisms of indirect effects}

If the optimal response of intermediate predators to an increased basal prey density is to increase the foraging effort, then an increase in basal prey density will make intermediate predators more active, which makes it easier for top predators to capture intermediate predators.

When the optimal foraging response of intermediate predators increasing basal prey density is to decrease the foraging effort, the availability of intermediate predators to top predators decreases as the prey population increases, creating a negative indirect effects of prey on top predators. Hence, there are counteracting positive direct effects and negative indirect effects of prey on top predators, creating a density-dependent response (Table 1). When the top predator population size increases, foraging effort by the intermediate predators decreases. Hence, there is a positive trait-mediated indirect effect as well as a negative densitymediated indirect effect of top predators on prey.

\section{A specific example of nonlinear benefit functions}

To gain more insights into the model, we redefine $B_{N}$ as $b_{N}$ - $\left(1 /\left(\mathrm{A}_{\mathrm{N}} \mathrm{C}_{\mathrm{N}} \mathrm{R}\right)\right)$, and $\mathrm{B}_{\mathrm{P}}$ as $\mathrm{b}_{\mathrm{P}}-\left(1 /\left(\mathrm{A}_{\mathrm{P}}\left(\mathrm{R}+\mathrm{C}_{\mathrm{N}} \mathrm{N}\right)\right)\right.$ in eqs (4)-(6) to obtain the following system of equations.

$$
\begin{aligned}
& \frac{d P}{d t}=P\left(b_{P}-\frac{1}{A_{P}\left(R+C_{N} N\right)}-m_{P}\right) \\
& \frac{d N}{d t}=N\left(b_{N}-\frac{1}{A_{N} C_{N} R}-C_{N} P-m_{N}\right) \\
& \frac{d R}{d t}=R\left(r\left(1-\frac{R}{K}\right)-C_{N} N-P\right)
\end{aligned}
$$

where logistic growth was assumed for basal prey.

By following the above procedure, the optimal foraging effort for this system is found to be

$$
\mathrm{C}_{\mathrm{N}}=\frac{1}{\sqrt{\mathrm{PA}_{\mathrm{N}} \mathrm{R}}}
$$

Table 1. Inter- and intra-specific effects. $U=N /\left(B^{\prime}{ }_{N} R^{2}\right), Z=\partial / \partial C_{N}\left(C_{N} B_{N}\right)$. The sign of $Z$ determines whether intermediate predators increase or decrease their foraging effort with respect to basal prey density. + (always positive), - (always negative), 0 (does not affect). When an expression is given, the sign of the expression determines the effect.

\begin{tabular}{llll}
\hline & Effect of R & Effect of $N$ & Effect of P \\
\hline${\text { Effect on } W_{\mathrm{R}}}^{\text {Effect on } \mathrm{W}_{\mathrm{N}}}$ & $\Phi^{\prime}+\mathrm{UZ}$ & - & $1+1 / \mathrm{U}$ \\
${\text { Effect on } \mathrm{W}_{\mathrm{P}}}^{+}$ & + & 0 & - \\
\hline
\end{tabular}


The equilibria for these eqs (8)-(10) are shown in Appendix 2 . Figure 2 illustrates per capita growth rates as functions of population density for each trophic level. This figure confirms the results of the general analysis described above.

\section{Effects of adaptive foraging on the stability of the IGP system}

To examine the effect of adaptive foraging on the stability of the community, a standard stability analysis was conducted by examining the Routh-Hurwitz criteria (May 1974). We compared results from the system with adaptive foraging effort (i.e. eq. 11) and the system with constant foraging effort $\left(C_{N}=1\right)$. The stability regions from both systems are indicated in Fig. 3. Clearly, adaptive foraging expands the stability regions (Fig. 3).

\section{Discussion}

The results shown here reinforce those of other studies demonstrating predator-induced trait-mediated indirect effects and the complex nature of higher-order interactions in IGP. The balance between costs and benefits of foraging activity leads to foragers exhibiting density-dependent adaptive foraging, which positively impact the prey population. Once such an interaction is generated because of the high connectance of IGP, it influences all other populations in the system. These indirect interactions exert strong effects which overrule the counteracting direct effects, thus emphasizing the importance of adaptive foraging behavior and associated indirect effects in population dynamics.

An unusual result observed in the IGP system is the top predator-basal prey relationship. These two species are found in the adjacent trophic levels and can hold all possible combination of relationships (i.e.,,,+++--+-- ). The same may not true if the system does not contain the IGP property. Because analyses used in this study are the same as those of Abrams (1992), they are easily comparable. In the linear food chain (Abrams 1992), all of interactions are defined by a definite sign specific to the cost and benefit functions. In the IGP system, the sign of the species interaction is not definite and is density-dependent, and a mutualism $(++)$ can occur depending on the densities of each species. Density-dependence arises because counteracting indirect effects are mediated by intermediate predators' foraging activity. Top predators eat basal prey (direct negative effect), and top predators eat and intimidate intermediate predators (indirect positive effects on basal prey). These two kinds of effects increase at different rates as top predator density increases, thus creating the resulting density-dependence. Without adaptive foraging, the counteracting positive effects may not be strong enough to overrule negative predation.

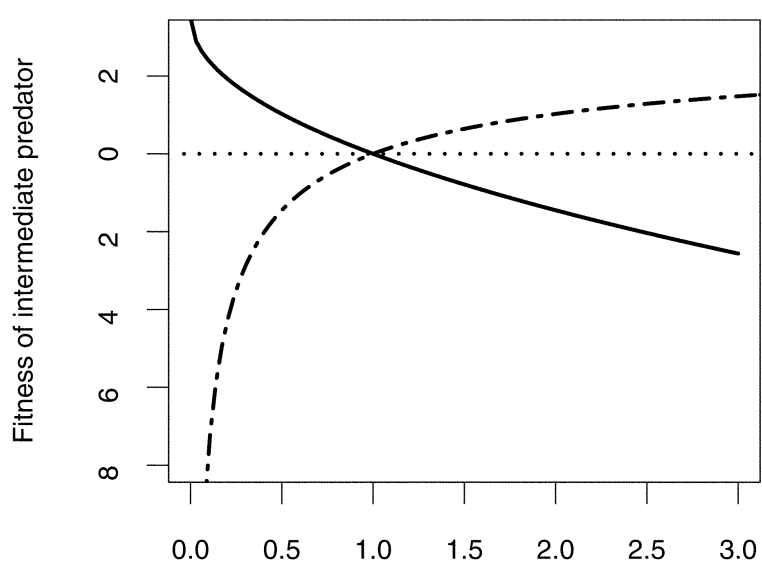

Population relative to equilibrium

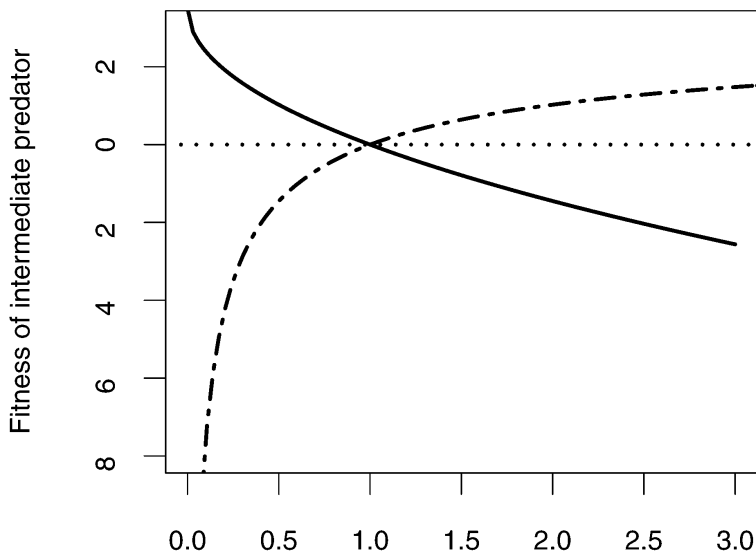

Population relative to equilibrium

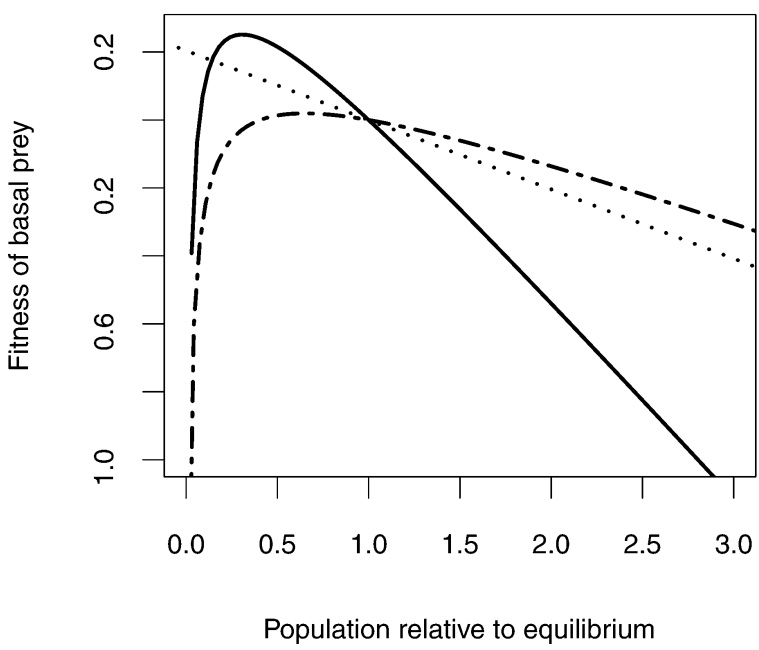

Fig. 2. Inter- and intra-specific effects of each species on other species. Top predators (solid line), intermediate predators (dotted line), basal prey (dashed line). $\mathrm{r}=1, \mathrm{~K}=1, \mathrm{~A}_{\mathrm{N}}=1, \mathrm{~A}_{\mathrm{P}}=1, \mathrm{~m}_{\mathrm{N}}=$ $0.5, \mathrm{~m}_{\mathrm{P}}=0.5, \mathrm{~b}_{\mathrm{P}}=3, \mathrm{~b}_{\mathrm{N}}=4$. 

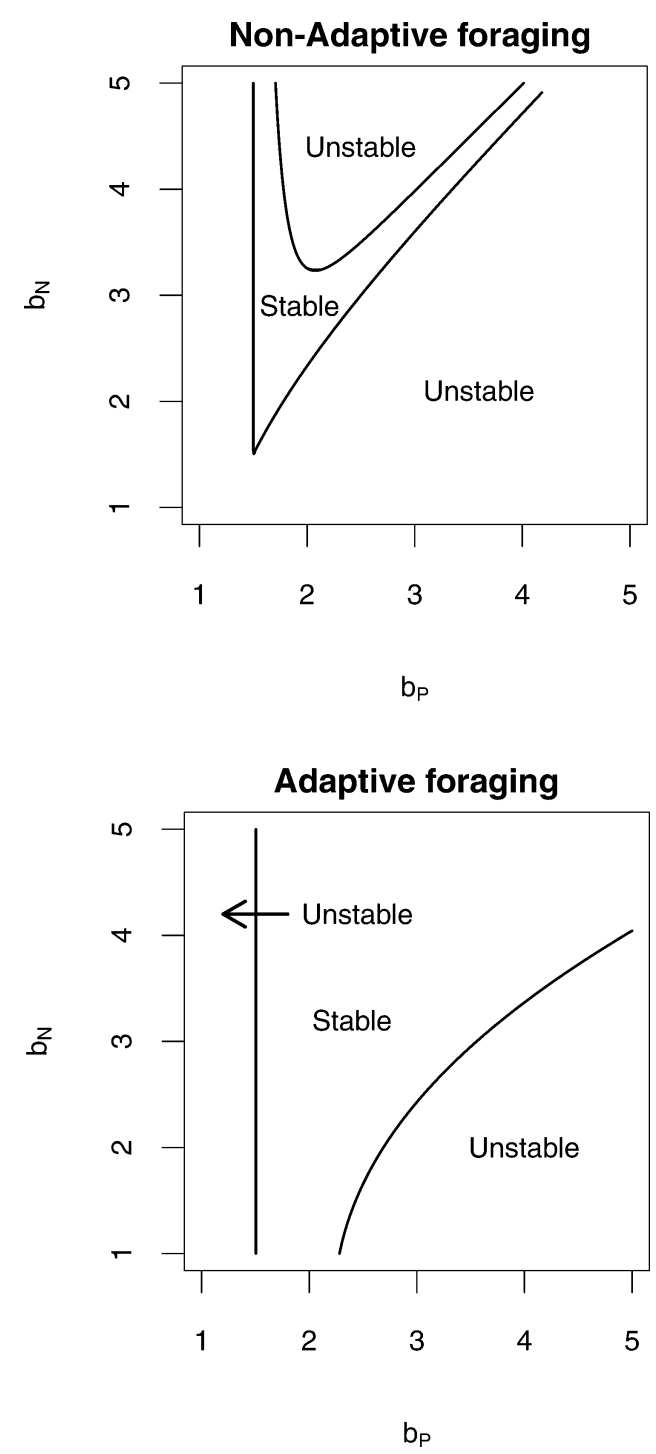

Fig. 3. An example of parameters $\left(b_{p}, b_{N}\right)$ that results in community stability. $\mathrm{r}=1, \mathrm{~K}=1, \mathrm{~A}_{\mathrm{N}}=1, \mathrm{~A}_{\mathrm{P}}=1, \mathrm{~m}_{\mathrm{N}}=0.5, \mathrm{~m}_{\mathrm{P}}=0.5$.

The stability analysis result suggests that adaptive foraging behavior may facilitate coexistence of the species. Because much of the instability of an IGP system seems to come from the exclusion of intermediate predators (Holt and Polis 1997), introducing the optimal foraging to intermediate predators resulting in enhancing the stability domain may seem trivial. Nevertheless, combined with widely documented antipredator behavior (e.g. Schultz 1981, Lima and Dill 1990, Okuyama 2002, Stoks et al. 2003), our result strengthens the hypothesis that such a behavior is a key explanatory factor for the IGP paradox. Furthermore, there are more effects associated with this adaptive behavior which are not captured in our model. For example, exclusion of top predators may be common when we introduce stochasticity and spatial explicitness to the sys- tem (e.g. individual based model) because when overall population levels are low, top predators may never encounter intermediate predators. This facilitates their extinction when top predators cannot reproduce sufficiently only from basal prey. In such a situation, intermediate predators increasing their foraging effort when top predator density is low will enhance the encounter rate and thus facilitate the survival of top predator species. In a scenario like this, adaptive foraging may help the persistence of the IGP system. Future researches in how the individual behavior interacts with stochasticity and space in community ecology are awaited.

Acknowledgements - We thank Tony Joern for encouraging us to pursue this problem. Comments from Kavita Isvaran improved the manuscript.

\section{References}

Abrams, P. A. 1989. Decreasing functional responses as a result of adaptive consumer behavior. - Evol. Ecol. 3: 95-114.

Abrams, P. A. 1992. Predators that benefit prey and prey that harm predators: unusual effects of interacting foraging adaptations. - Am. Nat. 140: 573-600.

Bolker, B. et al. 2003. Connecting theoretical and empirical studies of trait-mediated interactions. - Ecology 84: 1101-1114.

Borer, E. T. 2002. Intraguild predation in larval parasitoids: implications for coexistence. - J. Anim. Ecol. 71: 957-965.

Dinter, A. 1998. Intraguild predation between erigonid spiders, lacewing larvae and carabids. - J. Appl. Entomol. 122: 163167.

Fagan, W. F., Lukman, A. L. and Hartjahyo, H. 1998. Interactions between biological control efforts and insecticide application in tropical rice agroecosystems: the potential role of intraguild predation. - Biol. Control 13: 121-126.

Holt, R. D. and Polis, G. A. 1997. A theoretical framework for intraguild predation. - Am. Nat. 149: 745-764.

Johnson, D. M. 1993. Intraguild predation and cannibalism in odonate larvae: effects of foraging behavior and zooplankton availability. - Oikos 66: 80-87.

Kondoh, M. 2003. Foraging adaptation and the relationship between food-web complexity and stability. - Science 299: 1388-1391.

Křivan, V. 2000. Optimal intraguild foraging and population stability. - Theor. Popul. Biol. 58: 79-94.

Lima, S. L. and Dill, L. M. 1990. Behavioral decisions made under the risk of predation: a review and prospects. - Can. J. Zool. 68: 619-640.

Lucas, E., Coderre, D. and Brodeur, J. 1998. Intraguild predation among aphid predators: characterization and influence of extraguild prey density. - Ecology 79: 1084-1092.

May, R. M. 1974. Stability and complexity in model ecosystems. - Princeton Univ. Press.

Moran, M. D. and Hurd, L. E. 1994. Short-term responses to elevated predator densities: noncompetitive intraguild interactions and behavior. - Oecologia 98: 269-273.

Muller, C. B. and Brodeur, J. 2002. Intraguild predation in biological control and conservation biology. - Biol. Control 25: 216-223. 
Okuyama, T. 2002. The role of antipredator behavior in an experimental community of jumping spiders with intraguild predation. - Popul. Ecol. 44: 121-125.

Polis, G. A. and McCormick, S. J. 1987. Intraguild predation and competition among desert scorpions. - Ecology 68: 332-343.

Polis, G. A. and Holt, R. D. 1992. Intraguilds predation: the dynamics of complex trophic interactions. - Trends Ecol. Evol. 7: 151-154.

Polis, G. A., Myers, C. A. and Holt, R. 1989. The ecology and evolution of intraguild predation: potential competitors that eat each other. - Annu. Rev. Ecol. Syst. 20: 297-330.

Schmitz, O. J. 1998. Direct and indirect effects of predation and predation risk in old-field interaction webs. - Am. Nat. 151: 327-342.

Schultz, J. C. 1981. Adaptive changes in antipredator behavior of a grasshopper during development. - Evolution 35: 175-179.

Sergio, F., Marchesi, L. and Pedrini, P. 2003. Spatial refugia and the coexistence of a diurnal raptor with its intraguild owl predator. - J. Anim. Ecol. 72: 232-245.
Snyder, W. E. and Ives, A. R. 2003. Interactions between specialist and naturalist enemies: parasitoids, predators, and pea aphid biocontrol. - Ecology 84: 91-107.

Stoks, R., McPeek, M. A. and Mitchell, J. L. 2003. Evolution of prey behavior in response to changes in predation regime: damselflies in fish and dragonfly lakes. - Evolution 57: 574 585.

Suwa, M. 1986. Space partitioning among the wolf spider Pardosa amentata species group in Hokkaido, Japan. - Res. Popul. Ecol. 28: 231-252.

Szeinfeld, E. V. 1991. Cannibalism and intraguild predation in clupeoids. - Mar. Ecol. Progress Ser. 79: 17-26.

Werner, E. E. and Peacor, S. D. 2003. A review of trait-mediated indirect interactions in ecological communities. - Ecology 84: 1083-1100.

Wissinger, S. A. 1992. Niche overlap and the potential for competition and intraguild predation between size-structured population. - Ecology 73:1431-1444.

Appendix 1. Effects of population densities on fitness. The effects of population density on fitness are found by differentiating eq. (7) with respect to population densities. This requires the knowledge of how $\mathrm{C}_{\mathrm{N}}$ depends on population densities, which is found by implicit differentiation of eqs (4)-(6) with respect to the relevant population density. This results in the following list of formulae:

$$
\begin{array}{llrl}
\frac{\partial W_{R}}{\partial R}=\phi^{\prime}+\frac{N}{R}\left(\frac{B_{N}^{\prime}}{B^{\prime \prime}{ }_{N} R}+C_{N}\right) & \frac{\partial W_{R}}{\partial N}=-C_{N} & \frac{\partial W_{R}}{\partial P}=-\frac{N}{B^{\prime \prime}{ }_{N} R^{2}}-1 \\
\frac{\partial W_{N}}{\partial R}=\frac{P}{R} C_{N} & \frac{\partial W_{N}}{\partial N}=0 & \frac{\partial W_{N}}{\partial P}=-C_{N} \\
\frac{\partial W_{P}}{\partial R}=B_{P}^{\prime}\left(1-\frac{N}{R}\left(\frac{B_{N}^{\prime}}{B^{\prime \prime}{ }_{N} R}+C_{N}\right)\right) & \frac{\partial W_{P}}{\partial N}=C_{N} B_{P}^{\prime} & \frac{\partial W_{P}}{\partial P}=\frac{N_{B}^{\prime}}{B^{\prime \prime}{ }_{N} R^{2}}
\end{array}
$$

Appendix 2. Equilibrium densities. Equilibrium densities are determined by simultaneously solving the eqs (8)-(10). The formulae for equilibrium densities of each species are expressed as follows:

$\mathrm{P}^{*}=\frac{\mathrm{D}_{2} \mathrm{D}_{3}^{2}}{\mathrm{D}_{1} \mathrm{~A}_{\mathrm{N}}}$

$\mathrm{N}^{*}=8 \frac{\mathrm{D}_{2}}{\mathrm{D}_{1}^{2}} \mathrm{D}_{3}\left(\frac{\mathrm{D}_{1}}{4 \mathrm{~A}_{\mathrm{P}}\left(\mathrm{B}_{\mathrm{P}}-\mathrm{m}_{\mathrm{P}}\right)}-\mathrm{D}_{2}\right)$

$\mathrm{R}^{*}=4 \frac{\mathrm{D}_{2}}{\mathrm{D}_{1}}$

where

$\mathrm{D}_{1}=\mathrm{K}\left(\mathrm{A}_{\mathrm{N}}\left(\mathrm{B}_{\mathrm{N}}-\mathrm{m}_{\mathrm{N}}\right)^{2}-4\right)+4 \mathrm{r}$

$\mathrm{D}_{2}=\mathrm{rK}-\frac{\mathrm{K}}{\mathrm{A}_{\mathrm{p}}\left(\mathrm{B}_{\mathrm{P}}-\mathrm{m}_{\mathrm{p}}\right)}$

$\mathrm{D}_{3}=\mathrm{A}_{\mathrm{N}}\left(\mathrm{B}_{\mathrm{N}}-\mathrm{m}_{\mathrm{N}}\right)$ 